\title{
BMJ Open Is the Salut Programme an effective and cost-effective universal health promotion intervention for parents and their children? A register-based retrospective observational study
}

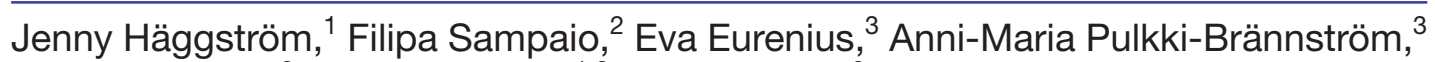
Anneli Ivarsson, ${ }^{3}$ Marie Lindkvist, ${ }^{1,3}$ Inna Feldman ${ }^{2}$

To cite: Häggström J, Sampaio F, Eurenius E, et al. Is the Salut Programme an effective and costeffective universal health promotion intervention for parents and their children? A register-based retrospective observational study. BMJ Open 2017;7:e016732. doi:10.1136/ bmjopen-2017-016732

- Prepublication history and additional material for this paper are available online. To view, please visit the journal (http:// dx.doi.org/10.1136/bmjopen2017-016732).

Received 7 March 2017 Revised 25 July 2017 Accepted 9 August 2017

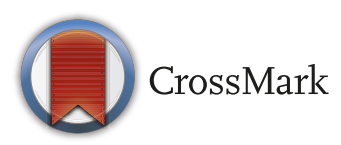

${ }^{1}$ Department of Statistics, Umeå School of Business and Economics, Umeå University, Umeå, Sweden

${ }^{2}$ Department of Public Health and Caring Sciences, Uppsala University, Uppsala, Sweden

${ }^{3}$ Department of Public

Health and Clinical Medicine, Epidemiology and Global Health, Umeå University, Umeå, Sweden

Correspondence to

Dr Inna Feldman;

inna.feldman@pubcare.uu.se

\section{ABSTRACT}

Objectives This study investigates the effectiveness and cost-effectiveness of the Salut Programme, a universal health promotion intervention, compared with care-as-usual, over the periods of pregnancy, delivery and the child's first 2 years of life.

Method We adopted a register-based retrospective observational design using existing data sources with respect to both exposures and outcomes. Health outcomes and costs were compared between geographical areas that received care-as-usual (non-Salut area) and areas where the programme was implemented (Salut area). We included mothers and their children from both the Salut and non-Salut areas if: (1) the child was born 2002-2004 (premeasure period) or (2) the child was born 2006-2008 (postmeasure period). The effectiveness study adopted two strategies: (1) a matched difference-in-difference analysis using data from all participants and (2) a longitudinal analysis restricted to mothers who had given birth twice, that is, both in the premeasure and postmeasure periods. The economic evaluation was performed from a healthcare and a limited societal perspective. Outcomes were clustered during pregnancy, delivery and birth and the child's first 2 years. Results Difference-in-difference analyses did not yield any significant effect on the outcomes. Longitudinal analyses resulted in significant positive improvement in Apgar scores, reflecting the newborn's physical condition, with more children having a normal Apgar score ( $1 \mathrm{~min}+3 \%$, $5 \mathrm{~min}+1 \%$ ). The cost of the programme was international dollar (INT\$)308/child. From both costing perspectives, the programme yielded higher effects and lower costs than careas-usual, being thus cost-saving (probability of around $50 \%$ ). Conclusions Our findings suggest that the Salut Programme is an effective universal intervention to improve maternal and child health, and it may be good value for money; however, there is large uncertainty around the cost estimates.

\section{BACKGROUND}

Development during the prenatal period, infancy and childhood is known to influence lifelong health, ${ }^{1-4}$ and the link between earlylife health and adult outcomes is strong and

\section{Strengths and limitations of this study}

- The findings suggest that the Salut Programme is an effective universal intervention to improve maternal and child health, and it may be good value for money.

- Our study contributes to the limited evidence base regarding universal multisectorial health promotion approaches during pregnancy and early childhood.

- A major strength of this study is that the 'state of the art' methods were used in the effectiveness analyses.

- Our analyses were limited to data available in registers. We lacked access to data on primary care and medication as well as on lifestyle and healthrelated quality of life.

- In the cost-effectiveness analyses, the limited societal perspective only included productivity losses due to mothers' inpatient and outpatient care, which might have contributed to the uncertainty in the results.

economically meaningful. ${ }^{5}$ Promotion of optimal child development and well-being comprises early detection and treatment of whole families, and it can potentially prevent the development of behavioural and emotional problems in children and adolescents. $^{6}$

Until now, the research community has failed to provide persuasive evidence about the effectiveness and cost-effectiveness of health promotion and preventive interventions. However, evaluation of intervention efforts is necessary for evidence-based decision-making. ${ }^{7} \quad 8$ Childhood obesity programmes have been suggested to be cost-effective, ${ }^{9}$ but other examples are rare. There are considerable methodological challenges when conducting such evaluations, and more thorough economic analyses of preventive programmes are encouraged. 
Economic evaluation is important for both those delivering and funding the interventions, ${ }^{10}$ and if demonstrated to be cost-effective, experiences and work modes can potentially be used in other settings.

The current project is nested within the Swedish Salut Child Health Intervention Programme, initiated in Västerbotten County in 2005 in addition to care-asusual. The programme is a multisectorial, family-centred approach to health promotion and prevention. One of the programme aims is avoidance of maternal and foetal pregnancy complications related to maternal lifestyle. This study aimed to investigate the effectiveness and cost-effectiveness of the Salut Programme compared with care-as-usual, over the periods of pregnancy, delivery and the child's first 2 years of life. The study was guided by the following research questions:

1. Does the Salut Programme improve maternal and child health?

2. What are the resource implications of the Salut Programme in terms of intervention and societal costs?

3. Is the Salut Programme a cost-effective public health intervention?

\section{METHODS}

\section{Overall study design and participants}

The current study adopted a register-based retrospective observational design using existing data sources with respect to both exposures and outcomes. ${ }^{11}$ We simulated an experiment by taking advantage of the stepwise implementation of the programme and nationally available individual-level register data collected independently of our study. ${ }^{12}$

Health outcomes and costs were compared between geographical areas that received care-as-usual (non-Salut area) and areas where the programme was implemented from 2006 and onwards (Salut area). The mother's place of residence at the child's birth determined whether the child and mother were classified as belonging to the Salut area or the non-Salut area. Thus, an intention-to-treat approach was used. ${ }^{13}$ We included mothers and their children from both the Salut area and non-Salut area if the child was born 2002-2004 (thus, before the Salut Programme was implemented anywhere), defined as the premeasure period. Accordingly, we included mothers and their children if the child was born 2006-2008 (thus, after the Salut Programme was implemented in some areas), defined as the postmeasure period. Henceforth, four study groups were formed: Salut pre, Salut post, non-Salut pre and non-Salut post.

We conducted an effectiveness study and an economic evaluation study. The effectiveness study adopted two complementary strategies: a matched difference-in-difference analysis using data from all participants and a longitudinal analysis restricted to the subsample of mothers who had given birth twice during the study period, both in the premeasure and postmeasure periods. The economic evaluation was conducted from both a healthcare and a limited societal perspective. In a recently published study protocol, we have described the Salut Programme and our planned analysis strategies. ${ }^{14}$ In the present study, this protocol has largely been followed. A few revisions have been made when necessary, and they are described and motivated below.

\section{Care-as-usual and the Salut Programme}

Care-as-usual during pregnancy and childhood is free of charge and decentralised to locally elected county councils with tax-raising powers, which creates some variation across the country in delivery of services. Almost all parents attend antenatal care, and likewise, almost all children attend child healthcare and dental care with an accompanying parent. Open preschools are free of charge, run by the municipality or churches and attended on a drop-in basis by families.

The Salut Programme is integrated within care-asusual, and it comprises strengthening and restructuring of care-as-usual and new specific interventions. Professionals in antenatal care, child healthcare, dental care and open preschools are invited to learning seminars and are encouraged to use manuals, specifically developed for the Salut Programme, to guide everyday practice. Following county-wide implementation, an evaluation showed significant improvements in professionals' health promotion practices and in collaboration across sectors. ${ }^{15}$ The programme is described in detail in online supplementary appendix A, tables A1 and A2and in previous publications. ${ }^{14-18}$

\section{Health outcomes}

Health outcome measures were chosen to demonstrate the performance of the Salut Programme with respect to supporting normal pregnancy and birth and, in other ways, contributing to the well-being of children and their mothers. Another prerequisite was that the measures were available through the Umea SIMSAM Lab, ${ }^{12}$ compiled from national and local registers. Moreover, we were guided by a recent publication on frequently measured outcomes to assess maternity care performance. ${ }^{19} \mathrm{~A}$ detailed description of the registers can be found elsewhere. ${ }^{14}$ The following time periods and outcome measures were chosen:

1. During pregnancy, delivery and at birth: mother's smoking status at first antenatal visit (yes/ no); pregnancy length at delivery $(\geq 37 /<37$ weeks); caesarean section (yes/no); birth weight $(\geq 2500 /<2500 \mathrm{~g})$; birth length $(\mathrm{cm})$; large for gestational age $(\geq 2 \mathrm{SD}$ above the reference population's mean weight); small for gestational age $(\leq 2 \mathrm{SD}$ below the reference population's mean weight); Apgar score 1, 5 and $10 \mathrm{~min}$ after delivery $(\geq 7 /<7$ points); child diagnosed by paediatrician as healthy (yes/no); and duration of mother's inpatient care related to delivery (days).

2. During the first 2 years after the child's birth: inpatient care not related to delivery within the two first months 


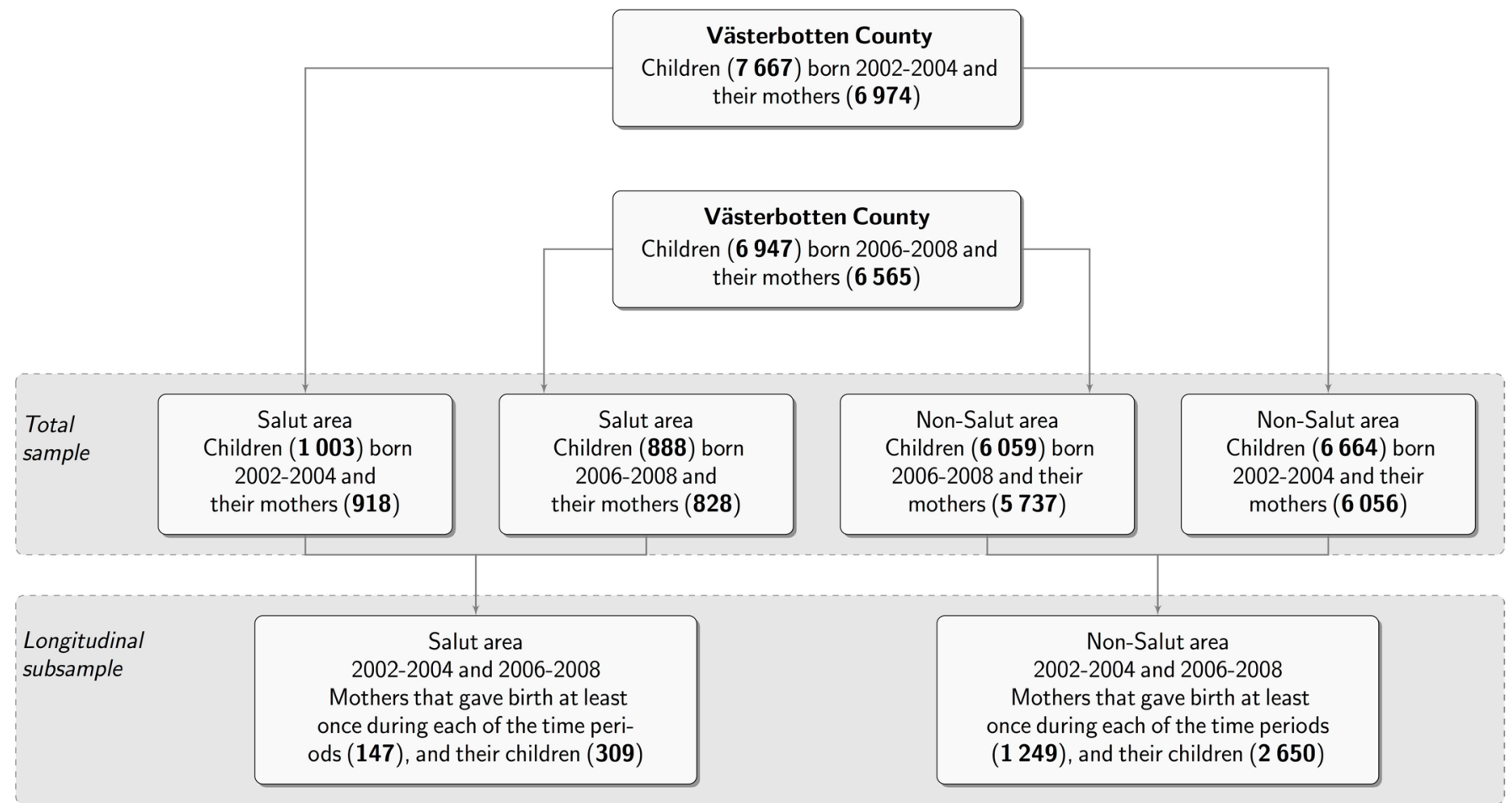

Figure 1 An overview of the study population and samples used in the analyses.

after child's birth (yes/no); cumulative duration of inpatient care (days); and cumulative number of outpatient visits, all for mother and child, respectively.

\section{Effectiveness analyses}

The samples are presented in figure 1. Assumptions and details regarding the analysis strategies are described elsewhere $^{14}$ and in online supplementary appendix B. The matched difference-in-difference analyses used the total sample. For each child born in the Salut area at postmeasure, matching observations were found in each of the other three groups: Salut area pre, non-Salut area pre and non-Salut area post. For every outcome, an observation was deemed a match if the mother, at the time of the child's birth, had the same level of education and similar age as the mother of a child born in the Salut area at postmeasure. The average difference over time in the Salut area was computed as the difference between the mean outcome in the Salut area at postmeasure and the mean outcome of the matched observations from the Salut area at premeasure. Analogously, the average difference over time in the non-Salut area was computed as the difference between the mean outcome of the matched observations from the non-Salut area at postmeasure and the mean outcome of the matched observations from the non-Salut area at premeasure. The final difference-in-difference estimate of the average treatment effect on the treated (ATT) was computed by subtracting the average difference over time in the non-Salut area from the average difference over time in the Salut area. To obtain CIs reflecting the uncertainty around the ATT point estimates, SE were computed using non-parametric bootstrapping with 1000 replications. ${ }^{20}$
In the longitudinal analyses, we used the subsample of mothers that gave birth to at least one child in each of the time periods and living in the same geographical area over the whole time period (figure 1). For a given outcome of interest, focusing on this subsample allowed us to use the mother's premeasure outcome value as a covariate on which to match on, in addition to the matching variables used in the difference-in-difference analyses. The simple matching estimate of the ATT was computed as the difference between the mean outcome in the Salut area at postmeasure and the mean outcome of the matched observations from the non-Salut area at postmeasure. Abadie-Imbens $\mathrm{SE}^{21}$ were computed to obtain CIs reflecting the uncertainty around the ATT point estimates. The SE computation is based on estimation of the asymptotic variance of the simple matching estimator and is preferable to bootstrapping in this case since the latter would lead to inconsistent SE estimation. ${ }^{22}$

In all analyses, matching was performed separately for each outcome variable, namely, the identity of the match was not fixed across analyses. Analyses were conducted in R V.3.3.0 $0^{23}$ using the Matching package ${ }^{24}$ for matching and Abadie-Imbens SE.

\section{Economic evaluation}

The economic analysis aimed to capture both the healthcare and the wider societal costs and benefits of the Salut Programme for the first 2years of the children's lives and their mothers. Two perspectives were adopted: a healthcare perspective, consisting of intervention costs and other healthcare resources used by children and mothers, and a limited societal perspective, additionally including productivity losses associated with 
mothers' illness. ${ }^{25}$ Results are expressed in 2013 purchasing-power parity international dollars (Swedish kroner (Kr) 8.71=INT\$1) after adjusting for inflation using the gross domestic product deflator. ${ }^{27}$

\section{Intervention cost}

Programme costs were estimated between January 2005 and June 2010. We added the opportunity cost of professionals' time to attend learning seminars during 20052007 (online supplementary appendix C, table C1) Calendar year-based allocation rules for joint costs and the division between start-up and implementation were decided on retrospectively by the Salut Programme staff to capture the changing nature of activities over time (online supplementary appendix C, table C2). Intervention costs were discounted at an annual rate of $3 \%$.

\section{Healthcare and other societal costs}

Healthcare-related costs were derived from information on the use of healthcare resources external to the Salut Programme, such as maternal inpatient care related to delivery and children's and mothers' inpatient and outpatient care due to illness. All healthcare-related costs were calculated for the child's first 2 years. Productivity losses due to mothers' illness were included in the analysis conducted from a limited societal perspective. Productivity losses were calculated using the human capital approach, by multiplying time off work due to inpatient and outpatient care by the average gross salary (including social charges). The average number of parental benefit days during the first year is around 220 for women in Sweden. ${ }^{28}$ Therefore, mothers were assumed to be on parental leave during the first year after childbirth, hence productivity losses were estimated for year two only. Contrary to the planned analyses in the study protocol, ${ }^{14}$ care of a sick child compensations were excluded from the analysis, as these were only linked to the parent and not to a particular child. In addition, these costs can be considered transfer payments, thus including them would constitute double counting. Total costs were estimated by multiplying frequencies of resources by their respective unit cost. Costs incurred during year two were discounted at $3 \%$. The difference in healthcare and other societal costs was compared between the Salut Programme and care-as-usual and between premeasure and postmeasure using permutation tests. Unit costs used to value resource use are listed in online supplementary appendix $\mathrm{C}$, table C3.

\section{Cost-effectiveness analysis}

The economic framework of this study is a retrospective register-based cost-effectiveness analysis. We compared costs and outcomes of the Salut Programme to care-asusual, from a healthcare and a limited societal perspective, and calculated incremental cost-effectiveness ratios (ICERs). Deterministic cost-effectiveness was expressed as the cost per low-Apgar case prevented. For the probabilistic analysis, we used non-parametric bootstrapping with
1000 replications to obtain $95 \%$ CIs around the ICER and investigate the uncertainty around the ICER estimates. The bootstrap results are presented on a cost-effectiveness plane. We explored the probability that Salut is cost-effective compared with care-as-usual, subject to a range of possible maximum values that a decision maker would be willing to pay for an additional low-Apgar case prevented. Cost-effectiveness acceptability curves (CEAC) for the healthcare and the limited societal perspectives were generated by plotting these probabilities for a range of willingness-to-pay (WTP) values. CEACs are a recommended decision-making approach to dealing with uncertainty regarding the cost-effectiveness estimates and the maximum values decision makers would be willing to pay for these. A decision maker who knows their maximum WTP for an additional unit of health gain can use the CEAC to determine the strength of the evidence on the cost-effectiveness of an intervention. ${ }^{29}$ Bootstrapping and the CEACs were performed in Excel 2011.

\section{RESULTS}

\section{Characteristics of the study population}

In the Salut area, 1003 and 888 children were born in the premeasure and postmeasure period, respectively (figure 1). In the non-Salut area, 6664 and 6059 children were born in the premeasure and postmeasure period, respectively. There were 147 mothers that gave birth at least once to 309 children in the Salut area and 1249 mothers that gave birth at least once to 2650 children in the non-Salut area. Characteristics of the total sample are given in table 1 and for the longitudinal subsample in online supplementary appendix D, table D1. Mothers giving birth to children in the Salut area were on average younger and less educated compared with mothers in the non-Salut area. The differences in age and education between Salut post and non-Salut post on the one hand and between Salut post and non-Salut pre on the other hand were all statistically significant with $p$ values below 0.001 . Between Salut post and Salut pre, there were no significant differences in age and education ( $p$ values 0.78 and 0.30 , respectively). Missing values varied between measures (online supplementary appendix D, tables D2 and D3). Information on mother's education was missing for $2.1 \%-2.4 \%$ of the Salut area observations and $1.0 \%-1.1 \%$ of the non-Salut area observations. All outcomes at birth exhibited some missingness, with the largest proportion for the smoking variable $(10.4 \%$ in Salut area pre). Outcomes during the first 2 years after birth were all fully observed.

\section{Effectiveness analyses}

Before conducting the difference-in-difference analyses, observations with missing values on outcome and/ or matching variables were excluded. The analytical sample sizes differed between outcomes since exclusion of observations was done separately for each outcome (online supplementary appendix D, tables D2 and D3). 
Table 1 Characteristics of the participants in the total sample

\begin{tabular}{|c|c|c|c|c|}
\hline & \multicolumn{2}{|c|}{ Salut area* } & \multicolumn{2}{|c|}{ Non-Salut area* } \\
\hline & Pre† & Post† & Pret & Post† \\
\hline \multicolumn{5}{|l|}{ Participants } \\
\hline Children, n & 1003 & 888 & 6664 & 6059 \\
\hline \multicolumn{5}{|l|}{ Covariates } \\
\hline \multicolumn{5}{|l|}{ Mother's education, \% } \\
\hline Compulsory school & 11.0 & 11.3 & 7.5 & 7.5 \\
\hline Secondary school & 51.2 & 48.1 & 44.5 & 36.8 \\
\hline Higher education & 37.8 & 40.6 & 48.0 & 55.7 \\
\hline \multicolumn{5}{|l|}{ Health outcomes } \\
\hline Caesarean section $\ddagger$ (yes), \% & 17.2 & 18.1 & 16.4 & 16.4 \\
\hline Birth weightๆ ( $\geq 2500 \mathrm{~g}), \%$ & 94.8 & 96.9 & 96.5 & 96.4 \\
\hline Birth length 9 (cm), mean (SD) & $50.3(2.8)$ & $50.3(2.9)$ & $50.5(2.5)$ & $50.3(2.5)$ \\
\hline LGA $\prod^{\star *}$ (yes), \% & 3.6 & 3.8 & 4.4 & 3.4 \\
\hline SGAף†† (yes), \% & 2.5 & 2.5 & 1.7 & 1.9 \\
\hline \multicolumn{5}{|l|}{ 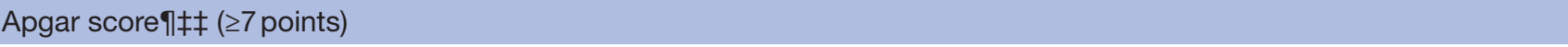 } \\
\hline At $1 \mathrm{~min}, \%$ & 95.8 & 96.3 & 95.3 & 94.6 \\
\hline At $5 \mathrm{~min}, \%$ & 99.1 & 99.4 & 98.7 & 98.5 \\
\hline Mother's inpatient careł††† (days), mean (SD) & $0.4(2.1)$ & $0.5(3.2)$ & $0.5(5.3)$ & $0.5(4.5)$ \\
\hline Child’s inpatient careๆ㧊 (days), mean (SD) & $1.9(12.8)$ & $1.5(8.2)$ & $1.5(8.1)$ & $1.4(9.6)$ \\
\hline 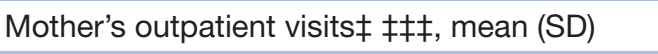 & $0.0(0.1)$ & $0.0(0.1)$ & $0.0(0.1)$ & $0.0(0.1)$ \\
\hline Child’s outpatient visits 㺻, mean (SD) & $0.0(0.2)$ & $0.1(0.4)$ & $0.0(0.2)$ & $0.1(0.7)$ \\
\hline
\end{tabular}

*Salut area, geographical area in Västerbotten county where the Salut Programme was implemented prior to 2009; non-Salut area, remaining part of Västerbotten county.

†Premeasure period, 2002-2004; postmeasure period, 2006-2008.

$\ddagger$ Outcome maternal health.

§Smoking status at first antenatal visit, around pregnancy week 12 .

ПOutcome child health.

** LGA, $\geq 2$ SD above the reference population's mean weight.

††SGA, $\leq 2 \mathrm{SD}$ below the reference population's mean weight.

$\ddagger \ddagger A$ measure of the newborn's physical condition 1, 5 and $10 \mathrm{~min}$ after birth, range 0-10.

$\S \S A$ healthy child according to a paediatrician's examination.

१११Mother's inpatient care related to delivery.

${ }^{\star * \star}$ Early inpatient care for mother and child, respectively, during the first 2 months after the child's birth but not related to the delivery. $\dagger \dagger+C$ umulative duration of inpatient care for mother and child, respectively, over the child's first 2 years, excluding care due to delivery complications.

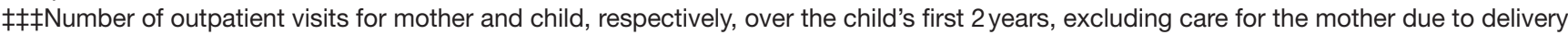
complications.

LGA, large for gestational age; SGA, small for gestational age. 
Table 2 Results of the effectiveness study, total sample and longitudinal subsample

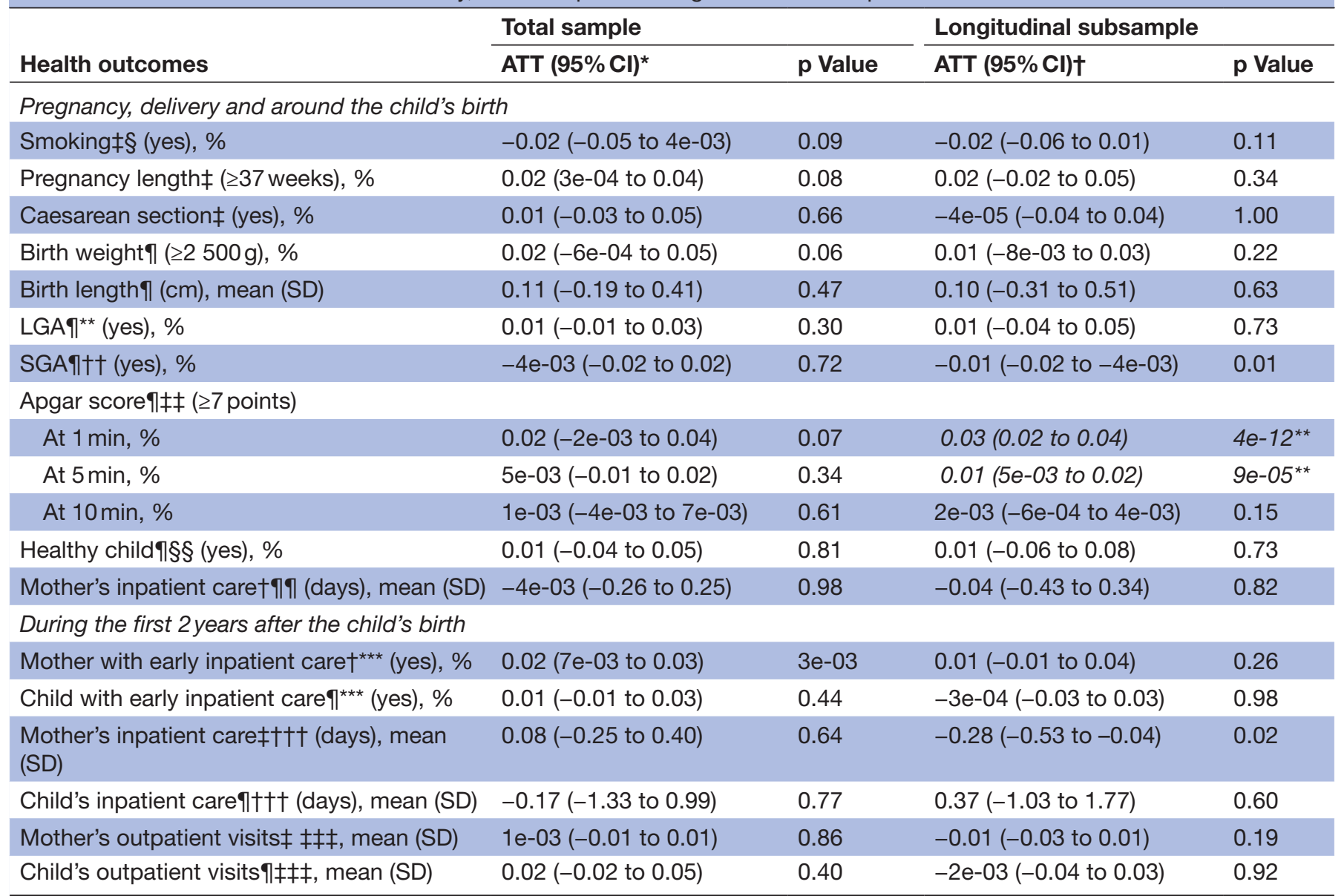

${ }^{*}$ Difference-in-difference estimates of the ATT with $95 \% \mathrm{Cls}$. Cls and $p$ values were computed with the assumption that ATT was normally distributed and with an SD equal to the bootstrap SE.

†Simple matching estimates of the ATT with 95\% (Cl).

fOutcome maternal health.

§Smoking status at first antenatal visit, around pregnancy week 12.

ๆOutcome child health.

**LGA, $\geq 2$ SD above the reference population's mean weight.

$\dagger † S G A, \leq 2$ SD below the reference population's mean weight.

$\ddagger \ddagger A$ measure of the newborn's physical condition 1, 5 and 10 min after birth, range 0-10.

$\S \S A$ healthy child according to a paediatrician's examination.

१ๆMother's inpatient care related to delivery.

${ }_{\star \star *}$ Early inpatient care for mother and child, respectively, during the first two months after the child's birth but not related to the delivery. $\dagger+\dagger C u m u l a t i v e$ duration of inpatient care for mother and child, respectively, over the child's first two years, excluding care due to delivery complications.

$\ddagger \ddagger \ddagger N$ umber of outpatient visits for mother and child, respectively, over the child's first two years, excluding care for the mother due to delivery complications.

**Statistically significant effect at the $\alpha=0.05$ level after a Bonferroni correction for multiple comparisons; ie, with the 38 outcome variables, this implies a significance threshold of $0.05 / 38=0.001$.

${ }^{* *}$ Statistically significant effect at the $\alpha=0.01$ level after a Bonferroni correction for multiple comparisons; ie, with the 38 outcome variables, this implies a significance threshold of $0.01 / 38=0.00026$.

ATT, average treatment effect on the treated; LGA, large for gestational age; SGA, small for gestational age.

The samples were well balanced before matching, but matching improved the covariate balance and resulted in standardised mean differences, ${ }^{30}{ }^{31}$ close to zero for all covariates in all analyses. The difference-in-difference analyses did not result in any significant ATT estimates. Hence, we conclude that for those individuals who were exposed to the Salut Programme, the programme had on average no significant effect on the outcomes studied (table 2), but the data suggest changes in a positive direction for the majority of health outcomes.

Before conducting the longitudinal analyses, the subsample of mothers giving birth at least once in each time period in the same area was further reduced in the following manner: for mothers who gave birth to more than one child in the same area at premeasure, observations from this period not relating to the last birth in that 
area and period were excluded. Analogously, if there were multiple births in the same area at postmeasure, observations from this period not relating to the first birth in that area and period were excluded. These exclusions were performed so that the variables at premeasure could be used as baseline variables to match on. Due to multiple births in the same area and period, observations were excluded from Salut area post (6), Salut area pre (9), non-Salut area post (49) and non-Salut area pre (103). Finally, observations with missing values on outcome and/ or covariates were excluded as in the difference-in-difference analyses (online supplementary appendix $\mathrm{D}$, tables D4 and D5). Matching improved the covariate balance and resulted in standardised mean differences close to zero for all covariates. The longitudinal analyses resulted in significant positive ATT estimates for the outcomes Apgar at 1 and 5 min (table 2).

We conclude that, for those who were exposed to the Salut Programme, in the subpopulation of mothers giving birth at least twice, there were 3\% (95\% CI: 2\% to $4 \%$ ) more births with high Apgar at 1 min compared with what would have been the case had they not been exposed to the programme. Similarly, there were $1 \%$ (95\% CI: $0.5 \%$ to 2\%) more births with high Apgar at 5 min compared with what would have been the case had they not been exposed to the Salut Programme. For our sample, this translates to 3.6 and 1.2 additional children having high Apgar at 1 and 5 min, respectively. We estimated the number needed to treat to prevent one case with low Apgar at $5 \mathrm{~min}$ by dividing one by the absolute risk reduction between Salut and non-Salut (0.02); 50 mothers would need to be exposed to the Salut Programme to prevent one case of low Apgar. The results for the other outcomes showed no significant effects; however, even for this population, we can see changes in a positive direction.

To assess how sensitive the results are to the exclusion of observations with missing values, analogous analyses were performed on samples where missing values had been imputed using multivariate imputations by chained equations with predictive mean matching. ${ }^{32}$ The results from analyses based on the samples with imputed values do not differ substantially from the results presented in table 2 and the conclusions that can be drawn are the same (online supplementary appendix D, table D6).

\section{Intervention costs}

The total cost of the Salut Programme was INT\$273 063 (Kr2 379 260). Averaged over the 888 children born in the Salut area at postmeasure gives a cost of INT $\$ 308$ (Kr2679) per child. The largest cost components were staff $(64 \%)$ and the opportunity cost of professionals' time to attend the learning seminars $(16 \%)$. Of the total, 28\% were start-up costs incurred during 2005-2007. The average annual implementation cost was INT\$43 575 (Kr379 677; averaged over 66 months).

\section{Healthcare and other societal costs}

Mean healthcare costs and productivity losses at premeasure and postmeasure for the Salut and the non-Salut areas for the longitudinal subsample $(\mathrm{n}=1289)$ are shown in table 3. Healthcare costs were lower in the Salut area due to less inpatient care for both mothers and children. Healthcare costs tended to be lower at postmeasure compared with premeasure in both areas, but the differences were not statistically significant. The SD around the mean healthcare cost estimates was large mostly because of large variation in inpatient care costs.

Productivity losses increased in the non-Salut area from premeasure to postmeasure $(+\mathrm{INT} \$ 29 ; \mathrm{p}=0.03)$, but it remained unchanged in the Salut area, which explains the difference in productivity losses over time in the Salut area compared with the non-Salut area (-INT\$31 per child; $\mathrm{p}=0.38$ ). Adding up healthcare costs and productivity losses, total costs (excluding intervention costs) were INT $\$ 1556$ lower at postmeasure than at premeasure in the Salut area and INT $\$ 1127$ lower at postmeasure than at premeasure in the non-Salut area. Hence, total costs fell by INT $\$ 430$ more per person in the Salut area compared with the non-Salut area $(\mathrm{p}=0.97)$. Analyses of healthcare costs and productivity losses for the total sample are found in the online supplementary appendix E, table E1.

\section{Cost-effectiveness analysis}

Both Apgar at 1 and 5 min showed statistically significant differences between Salut and non-Salut areas in the longitudinal analysis. Previous studies suggest that a low Apgar score at $5 \mathrm{~min}$ correlates with neonatal mortality and confers an increased risk of neurological disability and cognitive impairment. ${ }^{2633} 34$ In contrast, Apgar at $1 \mathrm{~min}$ is not a good predictor of infant outcomes. ${ }^{25}$ Hence, we considered Apgar at $5 \mathrm{~min}$ as the only relevant outcome in the cost-effectiveness analysis. The cost-effectiveness results for both costing perspectives are given in table 4 . From both a healthcare and a limited societal perspective, the Salut Programme yields higher effects and lower costs (ie, "dominant) than care-as-usual (non-Salut). The probability that the Salut Programme is cost-saving and entails positive effects compared with care-as-usual is approximately $50 \%$ ( $48.3 \%$ for the healthcare perspective and $49.7 \%$ for the limited societal perspective).

Figure E1 in the online supplementary appendix E presents the cost-effectiveness results on a cost-effectiveness plane for both costing perspectives. The bootstrapped estimates of incremental costs and effects fall approximately equally in the south-east and north-east quadrants of the plane. This is consistent with the Salut Programme having positive effects and an approximately $50 \%$ probability of being cost-saving compared with care-as-usual. The cost effectiveness plane demonstrates that the uncertainty around the cost estimates is indeed very large. This is further evidenced when plotting CEAC (Figure E2 in online supplementary appendix E) for different WTP values. With a zero WTP for preventing a case of 


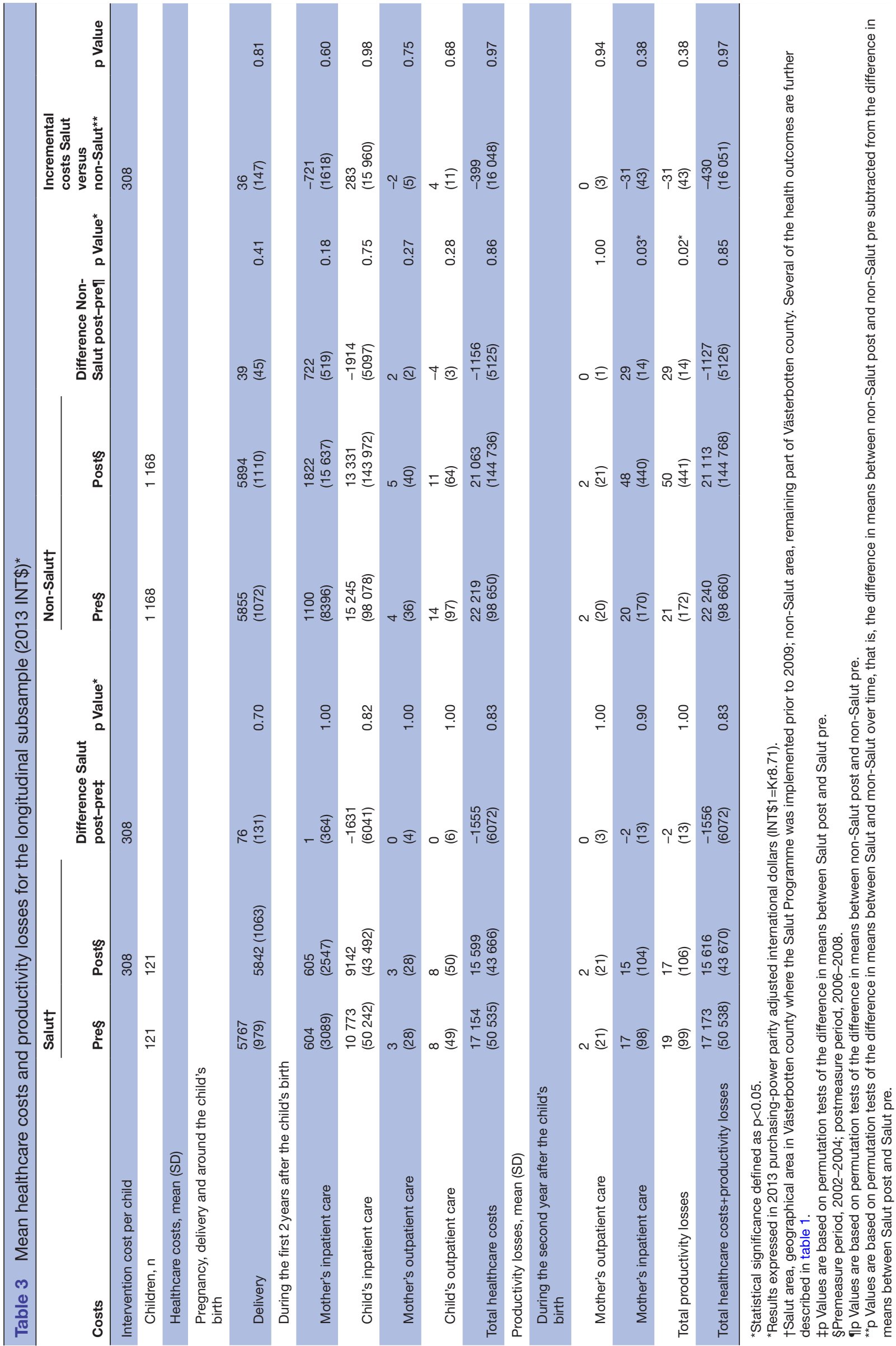


low Apgar, the probability that the Salut Programme is cost-effective is approximately $50 \%$. This probability hardly increases with WTP until very high ceiling values of INT $\$ 100000$ and above.

\section{DISCUSSION}

Main study findings and comparison with other studies

Our results suggest that the Salut Programme is an effective universal child health promotion intervention, and it is likely to represent good value for money. The difference-in-difference analyses did not show significant improvements in maternal and child health outcomes, but they suggested changes in a positive direction. However, the longitudinal analyses resulted in a significant positive improvement in Apgar scores, reflecting the newborn's physical condition, with more children having a normal Apgar score $(1 \mathrm{~min}+3 \%$, and $5 \min +1 \%)$. Notably, a recent publication suggests that a low Apgar score at $5 \mathrm{~min}$ may also serve as an indicator of poor maternal health. ${ }^{35}$ This recent published 6-year study of over 600000 newborns has extended the application of Apgar and identified a link between the newborn's Apgar score and the mother's need for intensive care.

The cost added by the programme to care-as-usual was small, INT\$308, representing only $4 \%$ of the average healthcare cost for the pregnancy, delivery and neonatal periods per woman/child, INT $\$ 7945 .{ }^{36}$ From both a healthcare and a limited societal perspective, the programme yielded higher effects and lower costs than care-as-usual, with approximately $50 \%$ probability of being cost-saving and entailing positive effects. Exploration of the uncertainty around the cost-effectiveness data showed that there was relatively large uncertainty around the cost estimates. In our view, the most likely explanation is that the noted differences in costs may not have been directly impacted by the intervention. Importantly, the Salut Programme would only have a higher probability of cost-effectiveness compared with care-as-usual if decision makers would be willing to pay much more (what seem unreasonably high financial figures) for an additional low-Apgar case prevented. Thus, our findings show that Salut can be good value for money. However, more evidence is needed about costs, in particular, how Salut may impact on healthcare costs in the long term.

Our study contributes to the limited evidence base regarding universal multisectorial health promotion interventions during pregnancy and early childhood. We are aware of only a few evaluations of the effectiveness and cost-effectiveness of such interventions. The universal parenting programme 'All Children in Focus', offered to parents of children aged 3 and above, showed a positive effect on parental self-efficacy and child health. ${ }^{37}$ However, the programme had a low probability of cost-effectiveness. ${ }^{38}$ Another study of a nurse-led intensive home visiting programme for first-time teenage mothers found no short-term benefits concerning the selected primary outcomes. $^{39}$ 


\section{Strengths and limitations of this study}

We evaluated the Salut Programme as it was implemented in current practice, which increases the external validity and generalisability of the results. The use of existing register data, in which exposure and outcomes have been routinely collected, ${ }^{12}$ reduces the amount of missing data. The 'state of the art' methods used in the effectiveness analyses, which do not require strong assumptions regarding the data generating mechanisms, allowed us to identify the differential effect of the programme on children and mothers born in Salut versus non-Salut areas in a natural experiment. ${ }^{40}$

While intention to treat $^{13}$ was the only feasible approach, we may have underestimated the intervention effects. We controlled for mothers' age and education using matching as well as the premeasure value of the outcome in the longitudinal analyses. However, we are aware of the risk for residual confounding. Another possible source of underestimation of effects is that the intervention development period (2005-2007) in part overlaps with the postmeasure period (children born 2006-2008). The retrospective study design limited us in terms of evaluating whether there was an initial learning period, during which effectiveness of the programme was lower. If such a learning period indeed existed, we may also have underestimated the opportunity cost of the programme because we assumed that (as stipulated by the programme) professionals integrated the programme interventions within care-as-usual. In the case visits that took more time than usual early on during implementation, a full societal perspective should also consider the incremental opportunity cost of parents' time. Due to the limitations of the retrospective design, we were not able to evaluate whether such a learning period existed.

As the Programme is a universal health promotion intervention, medical outcome measures were not expected to show significant effects. However, our analyses were limited to data available in registers. In particular, we lacked access to data on primary care visits and medication as well as on lifestyle and health-related quality of life. In the cost-effectiveness analyses, the limited societal perspective only included productivity losses due to mothers' inpatient and outpatient care, which might have contributed to the uncertainty in the results.

\section{Implications for policy and clinical practice}

Apgar scores have long been used as a measure for assessing infant well-being at birth, but 5 min Apgar scores in particular have also become a well-established predictive index for long-term outcomes such as neonatal morbidity and mortality in normal birth weighted infants. ${ }^{41-43}$ Low Apgar score at 5 min is associated with an increased risk of neurological disabilities. ${ }^{344}$ For example, $1.7 \%$ of newborns with low Apgar scores are diagnosed with cerebral palsy, compared with $0.05 \%$ of newborns with normal Apgar score at 5 min. ${ }^{45}$
Hence, to prevent one case of cerebral palsy, one would have to prevent 55 cases of low Apgar at $5 \mathrm{~min}$. As such, the estimated lifetime cost for a child with cerebral palsy is about INT $\$ 850000,{ }^{46}$ while the broad implementation of the Salut Programme would result in additional health benefits (cases of normal Apgar score) at no additional costs. Although there is no study estimating WTP for a low-Apgar case prevented, this comparison might serve as a reference frame.

Universal complex interventions implemented in reallife settings, such as the Salut Programme, are scarce and pose challenges with respect to implementation, dissemination and evaluation. ${ }^{47}$ The reliability of our results depends on how the Salut Programme was implemented in current praxis. Interviews with professionals suggest that key issues for effective implementation are involvement of professionals in intervention development, regular meetings with professionals and process consultants and the use of manuals. ${ }^{16}$ On the other hand, more resources would likely have improved feasibility by providing professionals with more dedicated time to deliver the interventions. Continuous support from decision makers is necessary ${ }^{48}$ to sustain the effectiveness and cost-effectiveness of an evidence-based intervention, such as the Salut Programme, in the long term.

\section{CONCLUSIONS}

Our study suggests that the Salut Programme is an effective universal intervention to improve maternal and child health, and it may be good value for money. The probability that the Salut Programme is cost-saving and entails positive effects is around $50 \%$ over a wide range of WTP ceiling values, although with a large uncertainty around the cost estimates.

Contributors The statistical analyses were carried out by $\mathrm{JH}$, and the economic evaluation was carried out by FS, A-MP-B and IF. Al and IF conceived and designed the study. $E E, M L$ and Al constitute the scientific steering group for the Salut Programme, and Al is principal investigator for the Umeå SIMSAM Lab, both prerequisites for the present study. All the authors (JH, FS, EE, A-MP-B, $\mathrm{Al}, \mathrm{ML}$ and IF) contributed to the writing process and have approved the final manuscript.

Funding This study was funded by the Swedish Research Council for Health, Working Life and Welfare (FORTE), grant number 2014-1399.

Competing interests None declared.

Ethics approval The Regional Ethical Review Board in Umeå gave clearance for the Salut Programme research (2010-14 63-31M) and for the Umeå SIMSAM Lab research (2010-157-310̈).

Provenance and peer review Not commissioned; externally peer reviewed.

Data sharing statement № additional data are avaliable.

Open Access This is an Open Access article distributed in accordance with the Creative Commons Attribution Non Commercial (CC BY-NC 4.0) license, which permits others to distribute, remix, adapt, build upon this work non-commercially, and license their derivative works on different terms, provided the original work is properly cited and the use is non-commercial. See: http://creativecommons.org/ licenses/by-nc/4.0/

(c) Article author(s) (or their employer(s) unless otherwise stated in the text of the article) 2017. All rights reserved. No commercial use is permitted unless otherwise expressly granted. 


\section{REFERENCES}

1. Forsdahl A. Are poor living conditions in childhood and adolescence an important risk factor for arteriosclerotic heart disease? $\mathrm{Br} J$ Prev Soc Med 1977;31:91-5.

2. Gluckman PD, Hanson MA. Living with the past: evolution, development, and patterns of disease. Science 2004;305:1733-6.

3. Kuh D, Ben-Shlomo Y. A life course approach to chronic disease epidemiology. Oxford University Press: Oxford, 2004.

4. Shonkoff JP, Boyce WT, McEwen BS. Neuroscience, molecular biology, and the childhood roots of health disparities: building a new framework for health promotion and disease prevention. JAMA 2009;301:2252-9.

5. Currie J, Rossin-Slater M. Early-life origins of life-cycle wellbeing: research and policy implications. J Policy Anal Manage 2015;34:208-42.

6. Weitzman C, Wegner L; Section on Developmental and Behavioral PediatricsCommittee on Psychosocial Aspects of Child and Family HealthCouncil on Early ChildhoodSociety for Developmental and Behavioral PediatricsAmerican Academy of Pediatrics. Promoting optimal development: screening for behavioral and emotional problems. Pediatrics 2015;135:384-95.

7. Nystrom ME, Hoog E, Garvare R, et al. Change and learning strategies in large scale change programs describing the variation of strategies used in a health promotion program. J Organ Change Manag 2013;26:1020-44.

8. Edwards RT, Charles JM, Lloyd-Williams H. Public health economics: a systematic review of guidance for the economic evaluation of public health interventions and discussion of key methodological issues. BMC Public Health 2013;13:1001.

9. John J, Wolfenstetter SB, Wenig CM. An economic perspective on childhood obesity: recent findings on cost of illness and cost effectiveness of interventions. Nutrition 2012;28:829-39.

10. Haynos AF, O'Donohue WT. Universal childhood and adolescent obesity prevention programs: review and critical analysis. Clin Psychol Rev 2012;32:383-99.

11. Berger ML, Martin BC, Husereau D, et al. A questionnaire to assess the relevance and credibility of observational studies to inform health care decision making: an ISPOR-AMCP-NPC Good Practice Task Force report. Value Health 2014;17:143-56.

12. Lindgren U, Nilsson $\mathrm{K}$, de Luna X, Luna de X, et al. Data Resource Profile: Swedish Microdata Research from Childhood into Lifelong Health and Welfare (Umeå SIMSAM Lab). Int J Epidemiol 2016;45:dyv358.

13. Gupta SK. Intention-to-treat concept: a review. Perspect Clin Res 2011:2:109-12.

14. Feldman I, Eurenius E, Häggström J, et al. Effectiveness and costeffectiveness of the Salut Programme: a universal health promotion intervention for parents and children-protocol of a register-based retrospective observational study. BMJ Open 2016;6:e011202.

15. Edvardsson K, Ivarsson A, Garvare R, et al. Improving child health promotion practices in multiple sectors-outcomes of the Swedish Salut Programme. BMC Public Health 2012;12:920.

16. Edvardsson K, Garvare R, Ivarsson A, et al. Sustainable practice change: professionals' experiences with a multisectoral child health promotion programme in Sweden. BMC Health Serv Res 2011;11:61.

17. Edvardsson K, Ivarsson A, Eurenius E, et al. Giving offspring a healthy start: parents' experiences of health promotion and lifestyle change during pregnancy and early parenthood. BMC Public Health 2011;11:936.

18. Eurenius $\mathrm{E}$, Lindkvist $\mathrm{M}$, Sundqvist $\mathrm{M}$, et al. Maternal and paternal self-rated health and BMI in relation to lifestyle in early pregnancy: the Salut Programme in Sweden. Scand J Public Health 2011;39:730-41.

19. Escuriet R, White J, Beeckman K, et al. Assessing the performance of maternity care in Europe: a critical exploration of tools and indicators. BMC Health Serv Res 2015;15:491.

20. Efron B. Bootstrap methods: another look at the jackknife. The Annals of Statistics 1979;7:1-26.

21. Abadie A, Imbens GW. Large sample properties of matching estimators for average treatment effects. Econometrica 2006;74:235-67.
22. Abadie A, Imbens G. On the failure of the bootstrap for matching estimators. Econometrica 2008;76:1537-57.

23. R: A Language and Environment for Statistical Computing [program]. Vienna, Austria: R Foundation for Statistical Computing, 2016.

24. Sekhon JS. Multivariate and propensity score matching software with automated balance optimization: the matching package for $R$. J Stat Softw 2011;42:1-52.

25. Casey BM, Mclntire DD, Leveno KJ. The continuing value of the Apgar score for the assessment of newborn infants. N Engl J Med 2001;344:467-71.

26. Ehrenstein V. Association of Apgar scores with death and neurologic disability. Clin Epidemiol 2009;1:45-53.

27. World Bank. World Development Indicators. http://data.worldbank. org (accessed 29 Nov 2016).

28. Försäkringskassan. 2012. Föräldrapenning: Analys av användandet 1974-2011.Social Insurance Report.

29. Gray AM, Clark PM, Wolstenholme JL, et al. Applied Methods of Cost-effectiveness Analysis in Health Care: Oxford University Press, 2011.

30. Rosenbaum PR, Rubin DB. Constructing a control-group using multivariate matched sampling methods that incorporate the propensity score. Am Stat 1985;39:33-8.

31. Stuart EA. Matching methods for causal inference: a review and a look forward. Stat Sci 2010;25:1-21.

32. Buuren Svan, Groothuis-Oudshoorn K. mice : Multivariate Imputation by Chained Equations in R. J Stat Softw 2011;45:1-67.

33. Bjørn AM, Jepsen P, Larsson HJ, et al. Hospitalizations for opioid poisoning: a nation-wide population-based study in Denmark, 19982004. Addiction 2009;104:104-8.

34. Thorngren-Jerneck K, Herbst A. Low 5-minute Apgar score: a population-based register study of 1 million term births. Obstet Gynecol 2001;98:65-70.

35. Ray JG, Medcalf KE, Park AL. Association of newborn Apgar score with maternal admission to the intensive care unit. JAMA Pediatr 2016;170:88-9.

36. de Keyser N, Josefsson A, Monfils WG, et al. Total cost comparison of standard antenatal care with a weight gain restriction programme for obese pregnant women. Public Health 2011;125:311-7.

37. Ulfsdotter M, Enebrink P, Lindberg L. Effectiveness of a universal health-promoting parenting program: a randomized waitlistcontrolled trial of All Children in Focus. BMC Public Health 2014; 14:1083.

38. Ulfsdotter M, Lindberg L, Månsdotter A. A cost-effectiveness analysis of the Swedish universal parenting program all children in focus. PLoS One 2015;10:e0145201.

39. Robling M, Bekkers MJ, Bell K, et al. Effectiveness of a nurse-led intensive home-visitation programme for first-time teenage mothers (Building Blocks): a pragmatic randomised controlled trial. Lancet 2016;387:146-55.

40. Bertrand M, Duflo E, Mullainathan S. How much should we trust differences-in-differences estimates? Q J Econ 2004;119:249-75.

41. Jepson HA, Talashek ML, Tichy AM. The Apgar score: evolution, limitations, and scoring guidelines. Birth 1991;18:83-92.

42. Montgomery KS. Apgar scores: examining the long-term significance. J Perinat Educ 2000;9:5-9.

43. Ehrenstein V, Pedersen L, Grijota M, et al. Association of Apgar score at five minutes with long-term neurologic disability and cognitive function in a prevalence study of Danish conscripts. BMC Pregnancy Childbirth 2009;9:14.

44. Stuart A, Otterblad Olausson P, Källen K. Apgar scores at 5 minutes after birth in relation to school performance at 16 years of age. Obstet Gynecol 2011;118:201-8.

45. Thorngren-Jerneck K, Herbst A. Perinatal factors associated with cerebral palsy in children born in Sweden. Obstet Gynecol 2006;108:1499-505.

46. Kruse M, Michelsen SI, Flachs EM, et al. Lifetime costs of cerebral palsy. Dev Med Child Neurol 2009;51:622-8.

47. Faria $\mathrm{R}$, Weatherly $\mathrm{H}$, Kiss $\mathrm{N}$, et al. The challenges in evaluating the cost-effectiveness of complex interventions. Value Health 2015;18:A727-A728.

48. Tibbits MK, Bumbarger BK, Kyler SJ, et al. Sustaining evidencebased interventions under real-world conditions: results from a largescale diffusion project. Prev Sci 2010;11:252-62. 